\title{
Prognostic significance of Tspan9 in gastric cancer
}

\author{
TONGTONG FENG**, LIBIN SUN*, WEIWEI QI, FEI PAN, JING LV, JING GUO, \\ SHUFEN ZHAO, AIPING DING and WENSHENG QIU
}

\begin{abstract}
Department of Oncology, Affiliated Hospital of Qingdao University, Qingdao, Shandong 266003, P.R. China
\end{abstract}
Received December 22, 2015; Accepted May 25, 2016

DOI: $10.3892 / \mathrm{mco} .2016 .961$

\begin{abstract}
Tetraspanins are a large superfamily of glycoproteins, which are engaged in a wide range of specific molecular interactions by forming tetraspanin-enriched microdomains. Tetraspanin 9 (Tspan9) is a previously poorly studied tetraspanin gene, which was predominantly identified as an amplified gene in serous Fallopian tube carcinoma. However, the expression and role of Tspan9 in gastric cancer have yet to be fully elucidated. The aim of the present study was to evaluate the expression and clinical significance of Tspan9 in gastric cancer. In the present study, 105 gastric cancer tissue samples and corresponding adjacent normal samples were detected for Tspan9 expression using immunohistochemistry; furthermore, the association between clinical characteristics and Tspan9 expression was also analyzed. Tspan 9 expression was determined to be significantly lower in cancer samples compared with those in corresponding adjacent normal samples $(\mathrm{P}<0.001)$. However, its increased levels of expression in cancer samples appeared to demonstrate a poorer prognostic tendency, which is associated with deeper tumor depth $(\mathrm{P}=0.025)$, more nodal involvement $(\mathrm{P}=0.01)$, more advanced tumor/lymph node/metastasis $(\mathrm{TNM})$ stages $(\mathrm{P}=0.017)$ and a larger tumor size $(\mathrm{P}=0.026)$. Additionally, multivariate analysis demonstrated that high expression of Tspan9 was an independent prognostic factor for poor overall survival $(\mathrm{P}<0.01)$. These results suggested that Tspan9 may be used as a potential prognostic factor in gastric cancer.
\end{abstract}

\section{Introduction}

Gastric cancer (GC) is a common cancer worldwide. Approximately 951,600 new patients were diagnosed with

Correspondence to: Professor Wensheng Qiu, Department of Oncology, Affiliated Hospital of Qingdao University, 16 Jiangsu Road, Qingdao, Shandong 266003, P.R. China

E-mail:wsqiuqd@163.com

\section{${ }^{*}$ Contributed equally}

Key words: Tspan9, tetraspanins, gastric cancer, immunhistochemistry, survival analysis, prognosis
GC, and 723,100 deaths occurred, in 2012; over $25 \%$ of the new cases occurred in Eastern Asia (1). With the development of mass screening for $\mathrm{GC}$, detecting malignant stomach lesions in the early stages has become a reality, and numerous patients with $\mathrm{GC}$ are now able to receive timely curative surgery or effective drug treatment (2). However, there remain a great number of patients who are diagnosed only in advanced stages, and thus have an unfavorable prognosis. Therefore, identifying and evaluating GC biomarkers is important for risk stratification, predicting survival and reducing mortality.

Tetraspanins, also called tetraspans or the transmembrane 4 superfamily (TM4SF), are a large family of evolutionarily conserved, four-transmembrane domain proteins (3). A burgeoning number of studies have discussed their involvement in tumor cell motility, invasiveness and the different aspects of the organization of tetraspanin microdomains (4-7). In 1999, seven new tetraspanins were identified from the EST database, named as new EST tetraspan NET-1 to NET-7 (8). The overexpression of NET-1 has been noted in human hepatocellular carcinoma, skin squamous cell carcinoma and cervical cancer (9-11). Upregulation of NET-6 has been demonstrated to be associated with a favorable outcome in prostate cancer (12). The previously poorly studied amplified gene, tetraspanin 9 (Tspan9; also known as NET-5) was predominantly found in serous Fallopian tube carcinoma (FTC) (13). The function of tetraspanins has not yet been fully elucidated, although their putative value as potential prognostic markers, including Tspan9, has been recognized.

To date, no studies have been published on the expression of Tspan9 in human GC tissue, and its clinical significance. In the present study, the expression of Tspan9 in GC tissues and adjacent non-cancerous tissues was detected by immunohistochemistry (IHC), and the association between the expression level of Tspan 9 and various clinicopathological characteristics was also investigated.

\section{Materials and methods}

Specimen source and patient information. A total of 105 patients with GC who had undergone radical gastrectomy in the Affiliated Hospital of Medical College, Qingdao University between April 2009 and November 2010 were enrolled in the present study. The samples were fixed in $10 \%$ formaldehyde solution and embedded in paraffin. None of the patients had received any preoperative treatment. 
Table I. Relationship between TSPAN9 expression and clinicopathological factors.

\begin{tabular}{|c|c|c|c|c|}
\hline Characteristics & No. & TSPAN9-lowa $(\mathrm{n}=55)$ & TSPAN9-high $^{\mathrm{a}}(\mathrm{n}=50)$ & P-value \\
\hline Gender & & & & 0.217 \\
\hline Male & 75 & 41 & 34 & \\
\hline Female & 30 & 14 & 16 & \\
\hline Age (year) & & & & 0.724 \\
\hline$<60$ & 36 & 18 & 18 & \\
\hline$\geq 60$ & 69 & 37 & 32 & \\
\hline Tumor differentiation & & & & 0.034 \\
\hline Well/moderate & 27 & 16 & 11 & \\
\hline Poor/no differentiation & 78 & 28 & 50 & \\
\hline Tumor size & & & & 0.026 \\
\hline$<5 \mathrm{~cm}$ & 56 & 35 & 21 & \\
\hline$\geq 5 \mathrm{~cm}$ & 49 & 20 & 29 & \\
\hline $\mathrm{T}$ factor & & & & 0.025 \\
\hline $\mathrm{T} 1 / \mathrm{T} 2$ & 54 & 34 & 20 & \\
\hline T3/T4 & 51 & 21 & 30 & \\
\hline Lymph node metastasis & & & & 0.01 \\
\hline Negative & 47 & 30 & 17 & \\
\hline Positive & 58 & 25 & 33 & \\
\hline TNM stage & & & & 0.017 \\
\hline $\mathrm{I} / \mathrm{II}$ & 65 & 40 & 25 & \\
\hline III & 40 & 15 & 25 & \\
\hline Lymphovascular invasion & & & & 0.312 \\
\hline Negative & 88 & 48 & 40 & \\
\hline Positive & 17 & 7 & 10 & \\
\hline Lauren's classification & & & & 0.199 \\
\hline Intestinal & 51 & 30 & 21 & \\
\hline Diffusion & 54 & 25 & 29 & \\
\hline Her-2 expression & & & & 0.04 \\
\hline Negative & 96 & 52 & 44 & \\
\hline Positive & 10 & 2 & 8 & \\
\hline
\end{tabular}

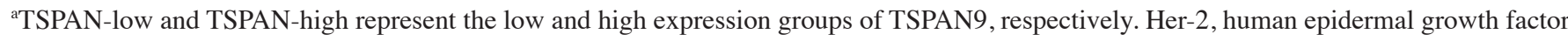
receptor 2; TSPAN9, tetraspanin 9; TNM stage, tumor/lymph node/metastasis stage.

IHC. The expression of Tspan9 was detected by IHC analysis with the SP method, using an SP-0023 Histostain-Plus kit (Beijing Biosynthesis Biotechnology Co., Ltd., Beijing, China). Rabbit monoclonal antibody against Tspan9 (rabbit anti-human; cat. no. ab106412) was purchased from Abcam, Inc. (Cambridge, MA, USA). The paraffin-embedded tissue blocks were sectioned in 3-4 $\mu \mathrm{m}$ slides and placed on glass slides (Jiangsu Huida Medical Instruments Co., Ltd., Jiangsu, China). For IHC staining, tissue sections were dewaxed in xylene and rehydrated with graded ethanol; subsequently, the slides were rinsed in phosphate-buffered saline (PBS). Antigen retrieval treatment was performed at $121^{\circ} \mathrm{C}$ for $2 \mathrm{~min}$ in $10 \mathrm{nmol} / 1$ sodium citrate buffer ( $\mathrm{pH}$ 9.0). Endogenous peroxidase was then inactivated by incubating with $0.3 \%$ hydrogen peroxide in methanol for $20 \mathrm{~min}$. Non-specific binding was blocked by treating the slides with normal goat serum fluid for $20 \mathrm{~min}$ at room temperature. Subsequently, the specimens were incubated with the anti-Tspan9 antibody (diluted $1: 100$ in PBS) at $37^{\circ} \mathrm{C}$ in an incubator for $1 \mathrm{~h}$. The sections were then washed three times with PBS ( 3 min each wash), and antibody binding was detected using the Histostain-Plus kit. Subsequently, the sections were washed three times ( 3 min each wash) with PBS, followed by the addition of diaminobenzidine as a chromogen. Positive control tissues were used to optimize the antibodies, according to the manufacturer's protocol. In negative controls, the primary antibody was replaced with PBS. According to the manufacturer's recommended criteria, the samples were independently scored for intensity of Tspan9 staining by two pathologists without knowledge of the clinical outcome at x100 and x200 magnification. The staining intensity was divided into three grades (using a scoring system of 0-3): No staining (0), slightly yellowish (1), brownish yellow (2) and dark-brown (3). The multiplications of the two scores were graded as follows: 0 (0 score), 1+ (1-4 score), $2+(5-8$ score $)$ and $3+(9-12$ score). Intensity scores of 0 or $1+$ 
A

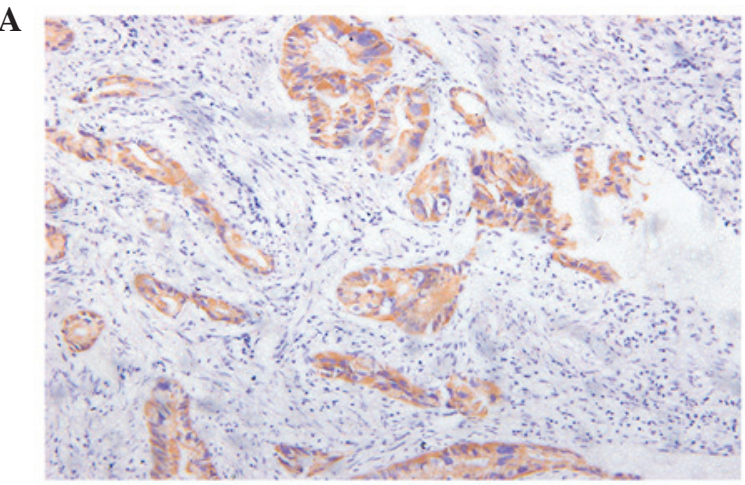

C

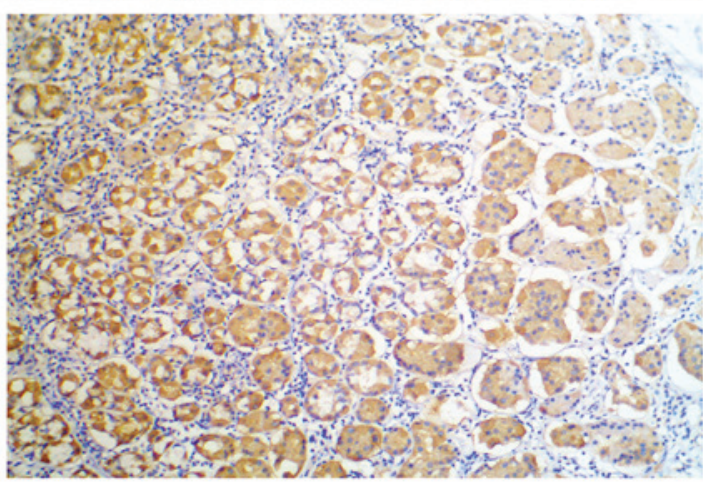

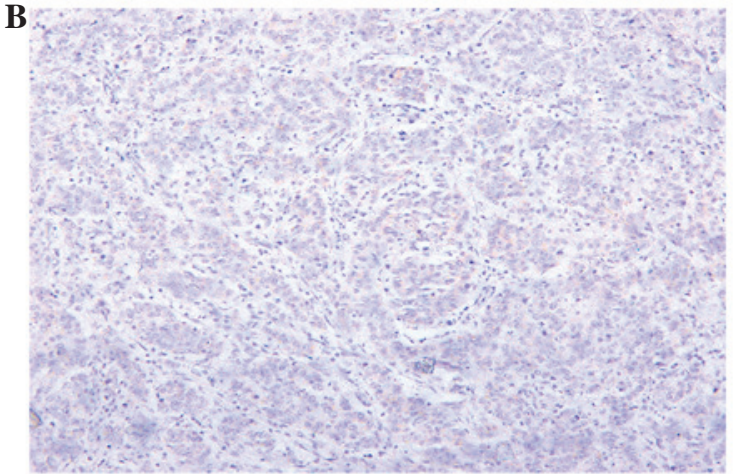

D

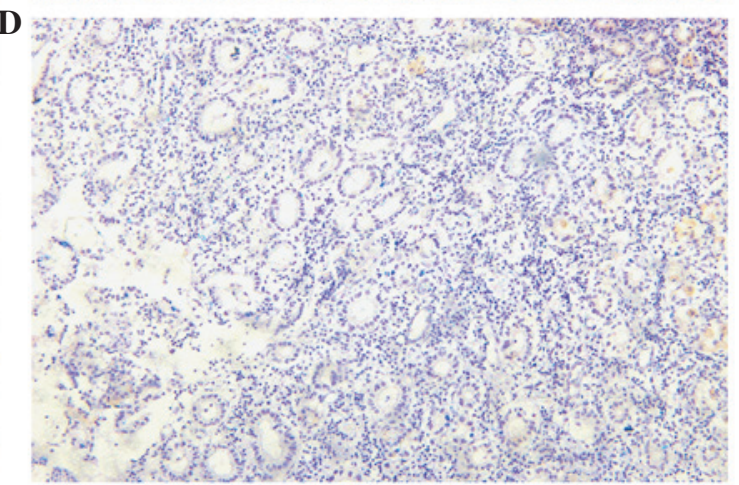

Figure 1. Tspan9 expression in GC and adjacent tissue identified by immunohistochemistry. Tspan9 was predominantly localized in the cytoplasm at different expression levels. (A) High expression of Tspan9 in GC tissues, (B) Low expression of Tspan9 in GC. (C) High expression of Tspan9 in adjacent normal tissue. (D) Negative expression of Tspan9 in adjacent tissue. (A-D) Magnification, x200. GC, gastric cancer; Tspan9, tetraspanin 9.

were designated as low expression, whereas those of $2+$ or $3+$ were designated as high expression.

Statistical analysis. The $\chi^{2}$ statistics test or Fisher's exact test were used to assess the correlation between Tspan9 expression and the clinicopathological characteristics. Overall survival curves were estimated using the Kaplan-Meier method, and the log-rank test was used to compare survival curves in the patient groups. A Cox proportional hazards model was used to examine the prognostic value of Tspan9 expression and other clinicopathological factors. Multivariate analysis was performed to determine the prognostic factors. $\mathrm{P}<0.05$ was considered to indicate a statistically significant value. The SPSS 17.0 statistical software program (SPSS, Inc., Chicago, IL, USA) was used for the analyses.

\section{Results}

Clinicopathological characteristics. The clinicopathological characteristics are shown in Table I. Of the patients, 75 were men and 30 were women, with a median follow-up period of 43 months (range 1-64 months) and a mean age of 53 years (range 27-75 years). The classification was based on the seventh edition of the tumor-lymph node-metastasis (TNM) staging system published by Biondi and coworkers (14). There were 33 cases with an extent of T1, 21 cases with T2, 8 cases with T3 and 43 cases with T4. There were 40 classified cases in stage I, 25 cases in stage II, 40 cases in stage III and 0 cases in stage IV. The histological type was poorly differentiated for 78 cases, moderately differentiated for 21 cases, and well differentiated for 6 cases.
Table II. TSPAN9 expression in GC tissues and normal tissues.

\begin{tabular}{lccc}
\hline $\begin{array}{l}\text { TSPAN9 } \\
\text { expression }\end{array}$ & $\begin{array}{c}\text { GC tissues } \\
(\mathrm{n}=105)\end{array}$ & $\begin{array}{c}\text { Normal tissues } \\
(\mathrm{n}=105)\end{array}$ & P-value \\
\hline Low & 55 & 19 & $\mathrm{P}<0.001$ \\
High & 50 & 86 & \\
\hline
\end{tabular}

GC, gastric cancer; TSPAN9, tetraspanin 9.

Tspan9 expression in GC and adjacent non-cancerous tissues. In the present study, sparse and partial Tspan 9 expression was identified in GC tissue (Fig. 1A and B) and adjacent non-cancerous tissues (Fig. 1C and D). High expression levels of Tspan9 were identified in 50/105 GC tissues, which was significantly decreased compared with that in adjacent non-cancerous tissues $(86 / 105 ; \mathrm{P}<0.001)$, as shown in Table II.

Correlation between Tspan9 expression and clinicopathological characteristics. The association of Tspan9 expression (high expression in 50 patients; low expression in 55 patients) with clinicopathological characteristics was subsequently analyzed. As shown in Table I, the Tspan9 high-expression group had a more advanced TNM stage $(\mathrm{P}=0.017)$, more nodal involvement $(\mathrm{P}=0.01)$, larger tumor sizes $(\mathrm{P}=0.026)$, a greater invasion factor $(\mathrm{P}=0.025)$, poorer tumor differentiation $(\mathrm{P}=0.034)$, and more positive expression levels of human epidermal growth factor receptor $2(\mathrm{Her}-2 ; \mathrm{P}=0.04)$ compared with the Tspan9-low group. However, no significant association was observed between the expression level of Tspan9 and 
Table III. Univariate and multivariate analysis of survival with clinicopathological factors.

Multivariate

analysis

Characteristic

Univariate P-value

HR $95 \%$ CI

P-value

\section{Gender}

Age (year)

Tumor differentiation

Tumor diameter

$\mathrm{T}$ factor

Lymph node metastasis

TNM stage

Lymphovascular invasion

Lauren's classification

Her-2 expression

Tspan9 expression
0.139

0.214

0.251

0.197

0.006

$<0.001$

$<0.001$

0.361

0.049

0.093

0.008
4.125

$<0.001$

2.025-8.405

1.522

$0.547-4.236$

1.951

0.966-3.942

1.754

0.731-7.208

2.166

$1.082-4.334$
0.422

0.062

0.208

0.029

HR, hazard ratio; CI, confidence interval; TNM, tumor/lymph node/metastasis; Her-2, human epidermal growth factor receptor 2; TSPAN9, tetraspanin 9 .
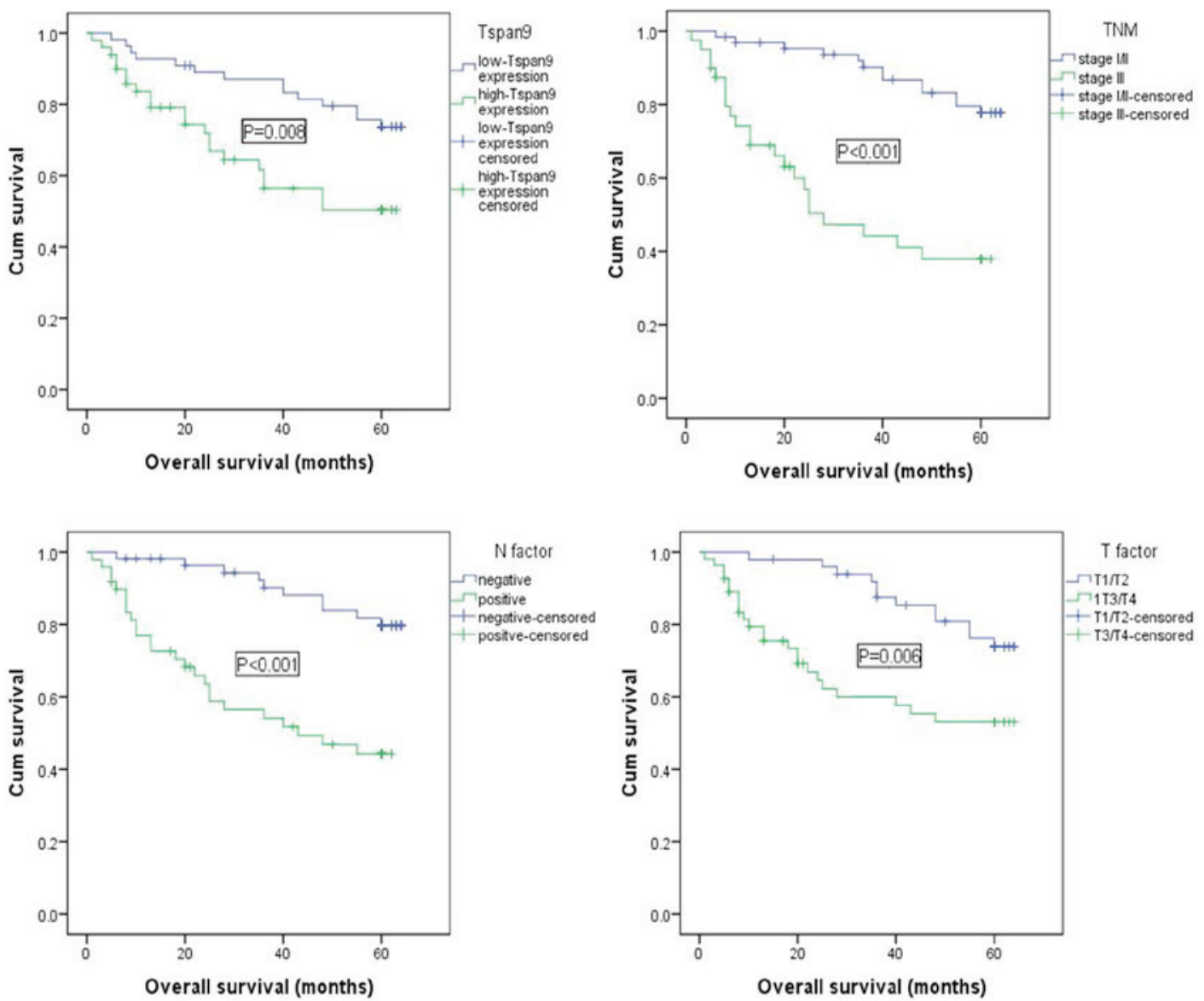

Figure 2. Kaplan-Meier survival analysis, revealing that patients with a high expression of Tspan9, positive lymph node metastasis, a greater T factor and advanced TNM stage had significantly worse overall survival rates. TNM, tumor/lymph node/metastasis; Tspan9, tetraspanin 9; Cum., cumulative. 
gender $(\mathrm{P}=0.217)$, age $(\mathrm{P}=0.724)$, lymphovascular invasion $(\mathrm{P}=0.312)$ or Lauren's classification $(\mathrm{P}=0.199)$.

Survival analysis of patients with GC according to Tspan 9 expression. The 4-year survival rate of the Tspan9 low-expression group was $78 \%$, which was significantly increased compared with the Tspan9 high-expression group $(38 \%$; $\mathrm{P}<0.01)$.

The association between the expression level of Tspan9 and prognosis. As shown in Fig. 2, univariate analyses, which were performed to estimate the clinical significance of prognostic factors that might influence the survival of the studied population, revealed that the TNM stage $(\mathrm{P}<0.001)$, Tspan9 expression $(\mathrm{P}=0.008)$, node metastasis $(\mathrm{P}<0.001)$ and $\mathrm{T}$ factor $(\mathrm{P}=0.006)$ were statistically significant risk factors affecting the overall survival of patients with GC. On the basis of the multivariate analysis data shown in Table III, Tspan9 expression was an independent prognostic marker for $\mathrm{GC}(\mathrm{P}=0.029)$, in addition to the TNM stage $(\mathrm{P}<0.001$; Table III).

\section{Discussion}

Tetraspanins are considered to function as 'organizers' in forming the type of specialized membrane domain known as a tetraspanin-enriched microdomain (15). Except for tetraspanins, the microdomains contain various adhesion receptors (e.g. integrins), transmembrane enzymes, immunoglobulin superfamily proteins and various other single and multi-transmembrane proteins (3). In terms of the function of tetraspanins, they are implicated in a number of cellular processes under physiological and pathological conditions, such as virus-induced syncytium, differentiation, cell adhesion, motility, tumor-cell metastasis, signal transduction, and so forth (16-20). Tetraspanins, including cluster of differentiation 9 (CD9) and Tspan24, have been associated with the prognosis of GC, as previously reported $(21,22)$.

Tspan9, a previously poorly studied tetraspanin molecule, drew our attention. Previously, our intention was to assay for the different levels of gene expression between drug-sensitive and drug-resistant cancer samples using gene microarray, and it was observed that the expression level of Tspan9 was increased in drug-resistant GC samples (data not shown). Therefore, at the outset of the present study our hypothesis was that Tspan9 was potentially a prognostic factor for chemoresistance, and that it may be associated with a poor prognosis of GC.

Tspan9 is one of TM4SF members with the $\mathrm{N}$ - and C-termini located in the cytoplasm, and it has been highly conserved during evolution (23). In 2013, a study published by Ooi et al (24) investigated host factors that were involved in alphavirus infection, and it was revealed that Tspan9 is critical for the efficient fusion of the virus in the endosome. Another previous study, which aimed to identify tetraspanin components in platelets, demonstrated that Tspan9 is a novel component of tetraspanin microdomains on the platelet surface (23). A study which aimed to investigate the detailed molecular interactions between primary serous ovarian carcinoma and FTC using a dedicated multiplex ligation-dependent probe amplification probe set identified the previously poorly studied gained/amplified gene, Tspan9, in FTC (13). To the best of our knowledge, no previous studies have demonstrated whether Tspan9 has a role in tumor progression.
The present study represents our first study on Tspan 9 expression in GC. In this study, Tspan9 expression was revealed to be markedly decreased in GC tissues compared with the adjacent non-cancerous tissues. However, it was of particular note that the overexpression of Tspan 9 was associated with a poor prognosis in GC. The 4-year survival rates in the Tspan9 high-expression group were clearly lower than those in the Tspan9 low-expression group, indicating that Tspan9 was a crucial factor in terms of clinical outcome in patients with GC. In the present study, the expression of Tspan9 was revealed to be an independent predictor of the overall survival rate. When the expression level of a factor in cancer tissue is lower than that in para-cancer tissue, it is more customary to associate the overexpression of the factor with a relatively good prognosis. The results obtained in the present study were therefore contrary to our expectations. In this study, the patients were divided into a Tspan9 low-expression group and a Tspan9 high-expression group, according to the results of the IHC. The association between the low or high expression of Tspan9 and clinicopathological features was then investigated. The results of the present study demonstrated that the high expression of Tspan9 was positively associated with an advanced TNM stage, lymph node metastasis, low differentiation, positive Her-2 expression and a diffuse Lauren classification, but was not associated with age, gender and lymphovascular invasion. Taken together it is possible to conclude that Tspan9 may contribute towards the development of GC.

In summary, Tspan9 is downregulated in GC, and it may be a useful prognostic factor for the survival of patients with GC. Tspan9 may exert important roles in the progression of GC. Further studies are required to investigate the mechanism of action, and the predictive value, of Tspan9 in GC.

\section{Acknowledgements}

This study was supported by the National Natural Science Foundation of China for mechanisms of tetraspanin Tspan9 in promoting 5-FU drug-resistance of gastric cancer through regulating autophagy in acidic tumors (no. 81472338) and a Project of the Shandong Province Higher Educational Science and Technology Program (no. J15LL58).

\section{References}

1. Torre LA, Bray F, Siegel RL, Ferlay J, Lortet-Tieulent J and Jemal A. Global cancer statistics, 2012. CA: A cancer journal for clinicians 65: 87-108, 2015.

2. Ishigami S, Arigami T, Uchikado Y, Setoyama T, Kita Y, Sasaki K, Okumura H, Kurahara H, Kijima Y, Harada A, et al: IL-32 expression is an independent prognostic marker for gastric cancer. Med Oncol 30: 472, 2013.

3. Romanska HM and Berditchevski F: Tetraspanins in human epithelial malignancies. J Pathol 223: 4-14, 2011.

4. Boucheix C, Duc GH, Jasmin C and Rubinstein E: Tetraspanins and malignancy. Expert Rev Mol Med 2001: 1-17, 2001.

5. Hemler ME: Tetraspanin functions and associated microdomains. Nat Rev Mol Cell Biol 6: 801-811, 2005.

6. Lazo PA: Functional implications of tetraspanin proteins in cancer biology. Cancer Sci 98: 1666-1677, 2007.

7. Zöller M: Tetraspanins: Push and pull in suppressing and promoting metastasis. Nat Rev Cancer 9: 40-55, 2009.

8. Serru V, Dessen P, Boucheix C and Rubinstein E: Sequence and expression of seven new tetraspans. Biochim Biophys Acta 1478: $159-163,2000$. 
9. Chen L, Wang Z, Zhan X, Li DC, Zhu YY and Zhu J: Association of NET-1 gene expression with human hepatocellular carcinoma. Int J Surg Pathol 15: 346-353, 2007.

10. Hölters S, Anacker J, Jansen L, Beer-Grondke K, Dürst M and Rubio I: Tetraspanin 1 promotes invasiveness of cervical cancer cells. Int J Oncol 43: 503-512, 2013.

11. Zhang J, Wang J, Chen L, Wang G, Qin J, Xu Y and Li X: Expression and function of NET-1 in human skin squamous cell carcinoma. Arch Dermatol Res 306: 385-397, 2014.

12. Arencibia JM, Martín S, Pérez-Rodríguez FJ and Bonnin A Gene expression profiling reveals overexpression of TSPAN13 in prostate cancer. Int J Oncol 34: 457-463, 2009.

13. Nowee ME, Snijders AM, Rockx DA, de Wit RM, Kosma VM, Hämäläinen K, Schouten JP, Verheijen RH, van Diest PJ, Albertson DG and Dorsman JC: DNA profiling of primary serous ovarian and fallopian tube carcinomas with array comparative genomic hybridization and multiplex ligation-dependent probe amplification. J Pathol 213: 46-55, 2007.

14. Biondi A and Hyung WJ: Seventh edition of TNM classification for gastric cancer. J Clin Oncol 29: :4338 -4339, 2011.

15. Boucheix C and Rubinstein E: Tetraspanins. Cell Mol Life Sci 58: 1189-1205, 2001.

16. Berditchevski $\mathrm{F}$ and Odintsova $\mathrm{E}$ : Characterization of integrin-tetraspanin adhesion complexes: Role of tetraspanins in integrin signaling. J Cell Biol 146: 477-492, 1999.

17. Boismenu R, Rhein M, Fischer WH and Havran WL: A role for CD81 in early T cell development. Science 271: 198-200, 1996.
18. Chambrion $\mathrm{C}$ and Le Naour F: The tetraspanins CD9 and CD81 regulate CD9P1-induced effects on cell migration. PloS One 5: e11219, 2010.

19. Charrin S, Latil M, Soave S, Polesskaya A, Chrétien F, Boucheix C and Rubinstein E: Normal muscle regeneration requires tight control of muscle cell fusion by tetraspanins CD9 and CD81. Nat Commun 4: 1674, 2013.

20. Detchokul S, Williams ED, Parker MW and Frauman AG: Tetraspanins as regulators of the tumour microenvironment: Implications for metastasis and therapeutic strategies. Br J Pharmacol 171: 5462-5490, 2014.

21. Yang H, Shen C, Zhang B, Chen H, Chen Z and Chen J: Expression and clinicopathological significance of CD9 in gastrointestinal stromal tumor. J Korean Med Sci 28: 1443-1448, 2013.

22. Yang YM, Zhang ZW, Liu QM, Sun YF, Yu JR and Xu WX Overexpression of CD151 predicts prognosis in patients with resected gastric cancer. PloS One 8: e58990, 2013.

23. Protty MB, Watkins NA, Colombo D, Thomas SG, Heath VL, Herbert JM, Bicknell R, Senis YA, Ashman LK, Berditchevski F, et al: Identification of Tspan9 as a novel platelet tetraspanin and the collagen receptor GPVI as a component of tetraspanin microdomains. Biochem J 417: 391-400, 2009.

24. Ooi YS, Stiles KM, Liu CY, Taylor GM and Kielian M: Genome-wide RNAi screen identifies novel host proteins required for alphavirus entry. PLoS Pathog 9: e1003835, 2013. 